\title{
Performance of a real-time PCR approach for diagnosing Schistosoma haematobium infections of different intensity in urine samples from Zanzibar
}

Dominique Keller ${ }^{1}$, Julian Rothen ${ }^{2,3}$, Jean-Pierre Dangy ${ }^{2,3}$, Corina Saner ${ }^{1}$, Claudia Daubenberger, ${ }^{2,3}$, Fiona Allan ${ }^{4}$, Shaali M. Ame ${ }^{5}$, Said M. Ali ${ }^{5}$, Fatma Kabole ${ }^{6}$, Jan Hattendorf ${ }^{2,3}$, David Rollinson ${ }^{4}$, Ralf Seyfarth ${ }^{1}$ and Stefanie Knopp $2,3^{*}$ (D)

\begin{abstract}
Background: Efforts to control and eliminate schistosomiasis have accelerated over the past decade. As parasite burden, associated morbidity and egg excretion decrease, diagnosis with standard parasitological methods becomes harder. We assessed the robustness and performance of a real-time PCR (qPCR) approach in comparison with urine filtration microscopy and reagent strip testing for the diagnosis of Schistosoma haematobium infections of different intensities.

Methods: The robustness of DNA isolation and GPCR was validated in eight laboratories from Europe and Africa. Subsequently, 792 urine samples collected during cross-sectional surveys of the Zanzibar Elimination of Schistosomiasis Transmission (ZEST) project in 2012-2017 were examined with qPCR in 2018. Diagnostic sensitivity of the qPCR was calculated at different infection intensity categories, using urine filtration microscopy as reference test. Spearman's rank correlation between Ct-values and S. haematobium egg counts was assessed and Ct-value percentiles for infection intensity categories determined.

Results: S. haematobium Dra1 DNA-positive samples were identified correctly in all eight laboratories. Examination of urine samples from Zanzibar revealed Dra1 DNA in 26.8\% (212/792) by qPCR, S. haematobium eggs in 13.3\% (105/792) by urine filtration, and microhaematuria in $13.8 \%$ (109/792) by reagent strips. Sensitivity of the GPCR increased with augmenting egg counts: 80.6\% (29/36) for counts between 1 and 4 eggs, 83.3\% (15/18) for counts between 5 and 9 eggs, 100\% (23/23) for counts between 10 and 49 eggs, and 96.4\% (27/28) for counts of 50+ eggs. There was a significant negative correlation between Ct-values and egg counts (Spearman's rho $=-0.49, P<0.001$ ). Seventy-five percent of the Ct-values were $\geq 33$ in the egg-negative category, $<31$ in the light intensity category, and $<24$ in the heavy intensity category.

(Continued on next page)
\end{abstract}

\footnotetext{
* Correspondence: s.knopp@swisstph.ch

${ }^{2}$ Swiss Tropical and Public Health Institute, Socinstrasse 57, 4002 Basel,

Switzerland

${ }^{3}$ University of Basel, Petersplatz 1, 4001 Basel, Switzerland

Full list of author information is available at the end of the article
}

\section{$\triangle B M C$}

(c) The Author(s). 2020 Open Access This article is licensed under a Creative Commons Attribution 4.0 International License, which permits use, sharing, adaptation, distribution and reproduction in any medium or format, as long as you give appropriate credit to the original author(s) and the source, provide a link to the Creative Commons licence, and indicate if changes were made. The images or other third party material in this article are included in the article's Creative Commons licence, unless indicated otherwise in a credit line to the material. If material is not included in the article's Creative Commons licence and your intended use is not permitted by statutory regulation or exceeds the permitted use, you will need to obtain permission directly from the copyright holder. To view a copy of this licence, visit http://creativecommons.org/licenses/by/4.0/ The Creative Commons Public Domain Dedication waiver (http://creativecommons.org/publicdomain/zero/1.0/) applies to the data made available in this article, unless otherwise stated in a credit line to the data. 
(Continued from previous page)

Conclusions: While the sensitivity of the qPCR was 80\% for very light intensity infections (egg counts $<10$ ), in general, the Dra1 based qPCR assay detected twice as many S. haematobium infections compared with classical parasitological tests. The qPCR is hence a sensitive, urine-based approach for $S$. haematobium diagnosis that can be used for impact assessment of schistosomiasis elimination programmes, individual diagnosis, and in improved format also for verification and certification of elimination.

Trial registration: ISRCTN, ISRCTN48837681. Registered 05 September 2012 - Retrospectively registered.

Keywords: Control, Diagnosis, Dra 1, Elimination, Microhaematuria, Real-time PCR, Schistosoma haematobium, Surveillance, Urine filtration, Zanzibar

\section{Background}

Schistosomiasis is a debilitating disease that particularly affects poor and economically deprived populations in 78 endemic countries worldwide [1]. In 2017, the global burden due to schistosomiasis was estimated at 1.43 million disability adjusted life years [2]. The World Health Organization (WHO) envisions a world free of schistosomiasis and urged their member states to scale up interventions with the goal to control the morbidity caused by infections with the blood fluke of the genus Schistosoma by 2020 , to achieve elimination as a public health problem by 2025 , and to interrupt transmission in selected countries by 2025 [1, 3, 4]. Over the past decade, efforts of schistosomiasis control programmes and drug donations accelerated, the number of people treated for schistosomiasis increased considerably, and in 2017, the coverage of school-aged children living in endemic countries targeted with praziquantel reached $68 \%$ [5].

There are several countries considered endemic for schistosomiasis that have successfully controlled morbidity, eliminated the disease as a public health problem, and even have interrupted transmission [6]. Most of these countries regularly treated infected individuals or the whole at-risk population for schistosomiasis, applied additional measures such as intermediate host snail control, environmental modification, health education and behaviour change interventions, and often experienced improvements of the socio-economic standard over time [6]. Moving from morbidity control towards elimination as public health problem, and finally interruption of transmission in endemic countries goes along with reductions in the prevalence and intensity of infection. As worm burdens decrease, fewer eggs are produced and excreted in urine (S. haematobium) or stool (other Schistosoma species). Lighter and asymptomatic infections are more difficult to diagnose and classical parasitological tests such as the reagent strip method to detect blood in urine as a proxy for $S$. haematobium infection or microscopy to detect Schistosoma eggs in urine or stool start to miss infections $[7,8]$. More sensitive diagnostic tests are required to monitor the impact of elimination efforts in areas where prevalence and infection intensity are low, for surveillance of potentially recrudescing transmission, and for verification of interruption of transmission [9-11].

Zanzibar is among the first settings in sub-Saharan Africa where elimination of urogenital schistosomiasis seems feasible and where elimination efforts implemented since 2012 have successfully reduced the prevalence and infection intensity to very low levels $[12,13]$.

Here, we assessed the robustness and performance of a standardized real-time polymerase chain reaction (qPCR) approach for the detection of the $S$. haematobium specific Dra1 DNA repeat in urine samples from Zanzibar. First, the robustness of DNA isolation from urine samples and the qPCR assay system were verified in a ring trial including a total of eight hospital, industry, and academic research laboratories from Europe and Africa. Subsequently, a selection of 792 urine samples from participants of the Zanzibar Elimination of Schistosomiasis Transmission (ZEST) project [14] that had been examined previously with reagent strips and urine filtration microscopy in Zanzibar between 2011 and 2017, were analysed by qPCR in Switzerland in 2018 and the performance of the qPCR system was evaluated. These selected samples represented a spectrum of egg counts to allow evaluation of the assay in relationship to intensity of infection.

\section{Methods \\ Study area}

Unguja and Pemba islands are the largest islands comprised in the Zanzibar archipelago offshore mainland Tanzania. The islands' projected population for 2019 was 1.6 million inhabitants [15].

Urogenital schistosomiasis caused by S. haematobium was highly endemic in the last century, with more than half of the school-aged population being infected in high-risk areas [16-18]. Intense morbidity control efforts with regular mass drug administration (MDA) campaigns implemented since the early 2000s and elimination interventions that started in 2011 with the ZEST project [14, 19-21], reduced the overall S. haematobium prevalence to $<2 \%$ and heavy infection intensities to $<1 \%$ in schoolchildren and adults in 2017 [12, 13]. 


\section{The ZEST project}

Efforts to eliminate urogenital schistosomiasis from Zanzibar started in 2011 with the formation of the ZEST alliance and strong political commitment from the Zanzibar government. The ZEST alliance included the Neglected Diseases Programme of the Zanzibar Ministry of Health $(\mathrm{MoH})$, the Public Health Laboratory-Ivo de Carneri (PHL-IdC), the WHO, the Schistosomiasis Control Initiative, the Bill \& Melinda Gates Foundation, the Schistosomiasis Consortium for Operational Research and Evaluation (SCORE), the Natural History Museum in London, the Swiss Tropical and Public Health Institute and other partners and stakeholders [14]. The Zanzibar $\mathrm{MoH}$ started biannual MDA of praziquantel in almost all communities and schools in Unguja and Pemba in 2012. In a cluster randomized trial funded by SCORE and implemented from 2011 to 2017, the impact of biannual MDA alone and in combination with snail control or behaviour change interventions was studied and compared [12-14]. One of the secondary objectives of the trial was to contribute to the validation and to assess the performance of new techniques for the diagnosis of S. haematobium.

\section{Field procedures}

Annual cross-sectional parasitological surveys were conducted in 91 study schools and 91 study communities, respectively, in Zanzibar (Pemba and Unguja islands), from November 2011 to May 2017. Every year and in each study location, urine samples were collected from $\sim 100$ randomly selected students aged 9-12 years and from $\sim 50$ randomly selected adults aged 20-55 years and examined for S. haematobium infections as described elsewhere in detail [12, 14, 21]. In brief, the head teacher of each study school was informed about the aim and purpose of the study and about the results of the previous study year in an annual meeting convened at the beginning of the school term. A few weeks later, each school was visited by the study team to register eligible children from grades 3 and 4, to randomly select $\sim 100$ participants, to invite them to participate in the study, to obtain their parents' written informed consent, and finally to collect their fresh urine samples for laboratory examination.

Similarly, the sheha (community head) of each study community (shehia) was informed about the study and subsequently the community was visited by the study team. Households were selected by a simple randomization procedure and a present adult household member was invited to participate in the study [14]. Once written informed consent was obtained, the participants were interviewed with a pretested questionnaire concerning demographic characteristics and MDA compliance, and invited to submit their own fresh urine sample.

All urine samples were collected between 10:00 AM and 2:00 PM and examined in the laboratory of the Zanzibar Neglected Diseases Programme in Unguja or at PHL-IdC in Pemba, respectively, during the later afternoon of the day of collection.

\section{Laboratory procedures}

Upon arrival in the laboratory, the urine samples were sorted by increasing participant ID and examined for microhaematuria using reagent strips (Hemastix; Siemens Healthcare Diagnostics Ltd., Camberley, United Kingdom). Microhaematuria was coded semiquantitatively in line with the colour reaction of the reagent strips and the manufacturer's instructions as negative; trace; +; ++; and +++. Subsequently, urine samples with sufficient volume were vigorously shaken, and 10 $\mathrm{ml}$ were filtered through a filterholder containing a polycarbonate filter with a pore size of $20 \mu \mathrm{m}$ (Sterlitech, Kent, WA, United States of America) using a standard $10 \mathrm{ml}$ plastic syringe. The filter was then carefully transferred to a microscope slide, which was labeled with the participant ID, covered with hydrophilic cellophane soaked in glycerol, stained with Lugol's iodine and examined under the microscope for the presence and number of $S$. haematobium eggs by trained laboratory technicians [7]. Finally, on the day of collection, $10 \mathrm{ml}$ of $10 \%$ of all urine samples were filled into Falcon tubes and stored in $\mathrm{a}-20^{\circ} \mathrm{C}$ freezer in Pemba for future use. Several hundreds of the frozen urine samples were transferred to Switzerland on dry ice for further examination with the qPCR approach described below.

\section{qPCR system \\ Sample selection}

A total of 1864 urine samples collected in Zanzibar between 2011 and 2017, were shipped on dry ice from Zanzibar to Basel in 2016 and 2017. Among them, 800 samples were selected by the study statistician for qPCR based examination at Biolytix AG in 2018, following these criteria: first, all samples from 2016 and 2017 were selected because there were only few samples available from those years; second, we selected all $S$. haematobium egg-positive samples and matched an egg-negative sample based on year, location, and survey (school/community) of collection. Finally, we randomly selected 585 samples to reach a total of 800 samples for examination with qPCR.

\section{Sample preparation for the ring trial}

For proficiency testing of the robustness of DNA isolation from urine and the Dral based qPCR, eight laboratories based in Europe and Africa were invited to test 
urine samples for S. haematobium Dra1 DNA in 2017. Two laboratories were based in sub-Saharan Africa (one in Tanzania and one in South Africa), and six in Europe (one in Germany, one in The Netherlands, one in Norway, two in Switzerland, and one in the United Kingdom). Among them, two were hospital laboratories, three were industry laboratories, and three were academic research laboratories. The aim of the ring trial was to explore how the DNA isolation and qPCR performed in different laboratories with different technicians and thermocyclers. Urine samples, the DNA Isolation Kit material, and the PCR Kit, respectively, were pooled and aliquoted at Biolytix AG before sending them to the ring trial participants to provide the same conditions for all participants. The urine samples (U1U8) were sent in duplicates: one egg-negative (U1 $\hat{=} \mathrm{U} 3$ ) and three $S$. haematobium egg-positive samples (U7 $\hat{=}$ U6, U2 $\hat{=} \mathrm{U} 4$, U5 $\hat{=} \mathrm{U} 8$ ). Hence, in total, eight samples were included in the test material. The participant laboratories were blinded to the infection status. In addition to the samples, one negative control (water as non-template control) and one positive control (sample containing Dra1 DNA), the Kit material and a detailed protocol for DNA isolation and examination were provided to the participants. Once the test samples and material were received, ring trial participants isolated DNA from the urine samples using the provided Quick-DNA Urine Kit (D3061) and protocol as described below. The isolated DNA was analyzed by the multiplex qPCR system described below. Each sample was run on a thermocycler in 1:10 dilution and undiluted. Results were assessed qualitatively for the presence or absence of DNA of the target organism, S. haematobium, and the reference gene $18 \mathrm{~S}$ rRNA. All participants submitted their qPCR results to Biolytix AG within three to 16 weeks. The results included the cycle threshold $(\mathrm{Ct})$ values of the qPCR analysis and the type of thermocycler used.

\section{DNA isolation and purification for $q P C R$}

DNA was extracted from the urine samples using the commercially available Quick-DNA Urine Kit (D3061) from Zymo Research according to the manufacturer's protocol for cellular and cell-free DNA precipitation, but using an elongated protein digestion time and using the Zymo-Spin I-96 Plate (C2004) instead of the Zymo-Spin IC-S Column.

First, for DNA precipitation, $4 \mathrm{ml}$ of each urine sample were mixed with $280 \mu \mathrm{l}$ of urine conditioning buffer and vortexed. Subsequently, $10 \mu \mathrm{l}$ of clearing beads were added and vortexed well. Finally, samples were spun for $15 \mathrm{~min}$ at $3000 \times \mathrm{g}$ in a Hermle Z366 Centrifuge (Machinenfabrik Berthold Hermle AG, Gosheim, Germany).
Second, for protein digestion, the supernatant was carefully discarded at low speed (leaving around 100 to $200 \mu \mathrm{l}$ liquid on top of the pellet). Subsequently, one volume of urine pellet digestion buffer was added to the mix. The pellet was then resuspended, and the mix suspended. Finally, $20 \mu \mathrm{l}$ of Proteinase $\mathrm{K}$ were added to the suspension and incubated for $1 \mathrm{~h}$ at $55^{\circ} \mathrm{C}$ in a shaking water bath. Third, for DNA purification, one volume of Genomic Lysis buffer was added to the digested mix $(420 \mu \mathrm{l})$ and vortexed. Subsequently, the mix was transferred in a new $2 \mathrm{ml}$ microcentrifuge tube, the sample was spun for $1 \mathrm{~min}$ at $3000 \times g$ in a Heraeus Pico 17 Centrifuge (Thermofisher Scientific, Waltham, United States of America), and the cleared supernatant was transferred into a new $2 \mathrm{ml}$ microcentrifuge tube. Then, the samples were transferred into a Zymo-Spin I-96 plate (C2004, Zymo Research, Irvine, United States of America) on top of a collection box and spun for $2 \mathrm{~min}$ at $5796 \times g$ in a Sigma $4-15 \mathrm{C}$ plate centrifuge (Qiagen, Hilden, Germany). The Zymo-Spin I-96 plate was placed onto a new collection block, $200 \mu \mathrm{l}$ of urine DNA prep buffer were added to each well with a multichannel pipette, and the plate was spun for $2 \mathrm{~min}$ at $5796 \times g$. Subsequently, the Zymo-Spin I-96 plate was placed onto a new collection block, $700 \mu \mathrm{l}$ of urine DNA wash buffer were added in each well with the multichannel pipette and the plate was spun for two minutes at $5796 \times g$. This step was repeated once, using $200 \mu \mathrm{l}$ of the same urine DNA wash buffer. Then, the plate was transferred on top of a 96-well elution tube rack and DNA was eluted with $100 \mu \mathrm{l}$ of DNA elution buffer prewarmed at $60^{\circ} \mathrm{C}$. Finally, the plate was spun for 2 $\min$ at $5796 \times g$ and the elution plate containing extracted DNA was covered with an adhesive aluminum sticker cover and labelled with a unique barcode number.

\section{Real-time PCR}

The primers used for the qPCR for detection of the Dral repeat unit in S. haematobium were selected based on the publication of Hamburger et al. 2001 [22]. To increase the sensitivity of the published PCR system from Hamburger et al. 2001 we used the probe published by Cnops et al. (2013) [23]. Moreover, the qPCR system was further optimized by adding an internal control detecting eukaryotic $18 \mathrm{~S}$ rRNA in a multiplex qPCR. The sequences for $S$. haematobium detection were as follows: Sh_F; 5'-GATCTCACCTATCAGACGAAAC-3', Sh_R 5'-TCACAACGATACGACCAAC-3', and Sh_P; 5 '-(FAM)-TGTTGGTGGAAGTGCCTGTTTCGCAABHQ1-3'. The sequences for the 18S rRNA detection were as follows: 18S_F; 5'-CGGCTACCACATCCAA GGAA-3', 18S_R; 5'-CTATTGGAGCTGGAATTA 
CCGC-3', and 18S_P; 5' -(YY)-TGCTGGCACCAGAC TTGCCCTCC-BHQ1-3'. The reaction was carried out in $20 \mu \mathrm{l}$ containing $1 \times$ Takyon ROX Probe MasterMix UNG (Eurogentec, Seraing, Belgium), 160 $\mathrm{nmol} / \mathrm{L}$ of Sh_F and Sh_R as well as $80 \mathrm{nmol} / \mathrm{L}$ of Sh_P, $40 \mathrm{nM}$ of $18 \mathrm{~S}-\mathrm{F}$ and $18 \mathrm{~S} \_\mathrm{R}$ as well as $20 \mathrm{nmol} /$ L 18S_P, and $4 \mu \mathrm{l}$ of target DNA. The participants of the ring trial used the thermocyclers available in their laboratory for the proficiency testing of the PCR system. The multiplex qPCR to examine the individual samples from Zanzibar for the second part of this study was carried out at Biolytix AG with an ABI 7900 HT thermocycler (ThermoFisher Scientific, Waltham, United States of America) with a 384 well block module using the thermal profile including 2 min at $50^{\circ} \mathrm{C}$ for the UNG activity, $10 \mathrm{~min}$ at $95^{\circ} \mathrm{C}$ followed by 50 cycles of $15 \mathrm{~s}$ at $95^{\circ} \mathrm{C}$, and $60 \mathrm{~s}$ at $60^{\circ} \mathrm{C}$.

\section{Data management and analysis}

The results of the macro- and microhaematuria grading and the number of $S$. haematobium eggs counted on the polycarbonate filter in $10 \mathrm{ml}$ urine were recorded on paper sheets by the laboratory technicians and entered into a Microsoft Excel (Microsoft Corporation 2010) database by data entry clerks in Zanzibar. The results of the qPCR examination were directly obtained in electronic format in Switzerland. Data were analysed using STATA version 14.0 (StataCorp., College Station, TX, USA). Only data from urine samples with complete microhaematuria, urine filtration, and $\mathrm{qPCR}$ result were included in the analyses.

Urine samples with a reagent strip colour reaction indicating trace, +, ++ or +++ were defined as microhaematuria-positive. Samples, where the single urine filtration microscopy revealed at least one $S$. haematobium egg per $10 \mathrm{ml}$ urine were considered as S. haematobium egg-positive. In line with WHO recommendations, S. haematobium infections were classified into light (1-49 eggs per $10 \mathrm{ml}$ urine) and heavy (50+ eggs per $10 \mathrm{ml}$ urine) intensity [1]. Additionally, to assess the performance of the qPCR at very low egg counts, we further stratified egg counts in the light infection intensity class into the following sub-classes: $1-5$ eggs $/ 10 \mathrm{ml}, 6-10$ eggs $/ 10 \mathrm{ml}, 11-49$ eggs $/ 10 \mathrm{ml}$. The DNA of each sample was diluted 1:2 and 1:20, respectively, and two Ct-value measurements per sample were taken for each dilution. The lowest $\mathrm{Ct}$-value among the measurements was taken into account for further analysis. Ct-values of $<33$ derived after 50 qPCR amplification cycles were considered as Dra1 DNA-positive. The sensitivity of the qPCR was calculated as the proportion of Dra1 DNA-positives that were correctly identified when compared with the results of the reference test. Reference tests, though imperfect since they lack sensitivity to detect very light intensity infection [7], were i) urine filtration microscopy, ii) reagent strip tests, and iii) combined results of urine filtration microscopy and reagent strip test. Sensitivity was also calculated stratified by the different egg count (sub-) classes to assess the performance of the qPCR at very light intensity infections. Correlation between $\mathrm{Ct}$-values derived after 50 amplification cycles of the qPCR and the number of $S$. haematobium eggs per $10 \mathrm{ml}$ urine were assessed with the Spearman's rank correlation test.

\section{Results}

Inter-laboratory robustness of qPCR

All eight laboratories that were invited did participate in the ring trial. The eight laboratories used five different types of thermocyclers for the qPCR (ABI 7500, ABI 7900HT, Agilent Technologies Stratagene MX3005P, Bio-Rad CFX96 Real-Time System, Roche LightCycler Nano).

As indicated in Table 1, all eight laboratories correctly identified the six S. haematobium egg-positive urine samples as Dra1 DNA-positive. Considering Ct-values of $\geq 33$ as negative, the two egg-negative samples were correctly identified by seven among the eight laboratories. One laboratory obtained Ct-values of 30.0 and 31.9, respectively, for these negative samples when diluted 1:10 and Ct-values of 26.7 and 28.1 for the undiluted samples, which we considered as an incorrectly positive result.

\section{Characteristics of urine samples from Zanzibar}

Urine samples from a total of 800 individuals from Zanzibar were selected for examination with qPCR in 2018 (Fig. 1). A total of 792 samples had complete examinations with urine filtration, reagent strips, and qPCR. Among the 212 samples with complete examinations collected in Unguja, 56 were from adults and 156 were from children. Among the 580 samples with complete examinations collected in Pemba, 156 were from adults and 424 were from children. S. haematobium eggs were identified by single urine filtration microscopy in $13.3 \%$, microhaematuria by reagent strips in $13.8 \%$, and S. haematobium DNA by qPCR in $26.8 \%$ of the urine samples.

\section{Sensitivity of qPCR with urine filtration microscopy and/ or reagent strips as reference method}

As indicated in Table 2 and Tab. S1, in total, the qPCR detected more than twice as many urine samples (212/ 792) as Schistosoma-positive than the urine filtration (105/792) and the reagent strips (109/792), respectively. Among the 212 Dra1 DNA-positive samples, 44.3\% (94/ $212)$ were egg-positive, $40.6 \% \quad(86 / 212)$ were microhaematuria-positive, and $48.1 \%(102 / 212)$ were either egg-positive or microhaematuria-positive. 
Table 1 Cycle threshold (Ct)-values of the qPCR analysis performed by different laboratories to detect S. haematobium Dra1 DNA in eight different urine sample pools, respectively

\begin{tabular}{|c|c|c|c|c|c|c|c|c|c|}
\hline Code & (1:10 dilution Dra1) & Laboratory 1 & Laboratory 2 & Laboratory 3 & Laboratory 4 & Laboratory 5 & Laboratory 6 & Laboratory 7 & Laboratory 8 \\
\hline$\overline{\mathrm{U} 1}$ & $\begin{array}{l}\text { Urine, Schistosoma } \\
\text { egg-negative }\end{array}$ & no-amp & no-amp & no-amp & no-amp & no-amp & 0.0 & 37.7 & 30.0 \\
\hline U2 & $\begin{array}{l}\text { Urine, Schistosoma } \\
\text { egg-positive }\end{array}$ & 21.5 & 20.4 & 22.6 & 19.2 & 20.4 & 21.6 & 21.3 & 18.2 \\
\hline U3 & $\begin{array}{l}\text { Urine, Schistosoma } \\
\text { egg-negative }\end{array}$ & 47.5 & no-amp & no-amp & no-amp & no-amp & 0.0 & 36.9 & 31.9 \\
\hline U4 & $\begin{array}{l}\text { Urine, Schistosoma } \\
\text { egg-positive }\end{array}$ & 21.4 & 20.7 & 21.2 & 18.5 & 19.3 & 22.7 & 20.7 & 19.7 \\
\hline U5 & $\begin{array}{l}\text { Urine, Schistosoma } \\
\text { egg-positive }\end{array}$ & 20.4 & 22.2 & 22.3 & 22.9 & 26.0 & 19.5 & 22.1 & 24.5 \\
\hline U6 & $\begin{array}{l}\text { Urine, Schistosoma } \\
\text { egg-positive }\end{array}$ & 23.6 & 18.8 & 18.8 & 19.1 & 18.6 & 22.0 & 19.8 & 20.8 \\
\hline U7 & $\begin{array}{l}\text { Urine, Schistosoma } \\
\text { egg-positive }\end{array}$ & 18.6 & 18.6 & 21.1 & 16.3 & 17.3 & 19.4 & 20.6 & 20.2 \\
\hline U8 & $\begin{array}{l}\text { Urine, Schistosoma } \\
\text { egg-positive }\end{array}$ & 25.7 & 27.0 & 28.1 & 25.5 & 26.6 & 24.8 & 27.7 & 27.6 \\
\hline PC & Positive Control & 22.6 & 24.6 & 23.2 & 21.9 & 23.3 & 20.13 & 27.4 & 23.7 \\
\hline \multirow[t]{2}{*}{ NTC } & No Template Control & no-amp & no-amp & 38.3 & no-amp & no-amp & no-amp & no-amp & no-amp \\
\hline & Thermocycler & Abi7900 & ABI 7500 & $\begin{array}{l}\text { Bio-rad } \\
\text { CFX } 96\end{array}$ & $\begin{array}{l}\text { Stratagene } \\
\text { MX3005P }\end{array}$ & Bio-rad CFX 96 & Bio-Rad CFX 96 & $\begin{array}{l}\text { Bio-Rad } \\
\text { CFX } 96\end{array}$ & $\begin{array}{l}\text { Roche } \\
\text { LightCycler } \\
\text { Nano }\end{array}$ \\
\hline
\end{tabular}

no-amp No DNA amplification up to cycle 50 . Ct-values of $\geq 33$ were considered as negative

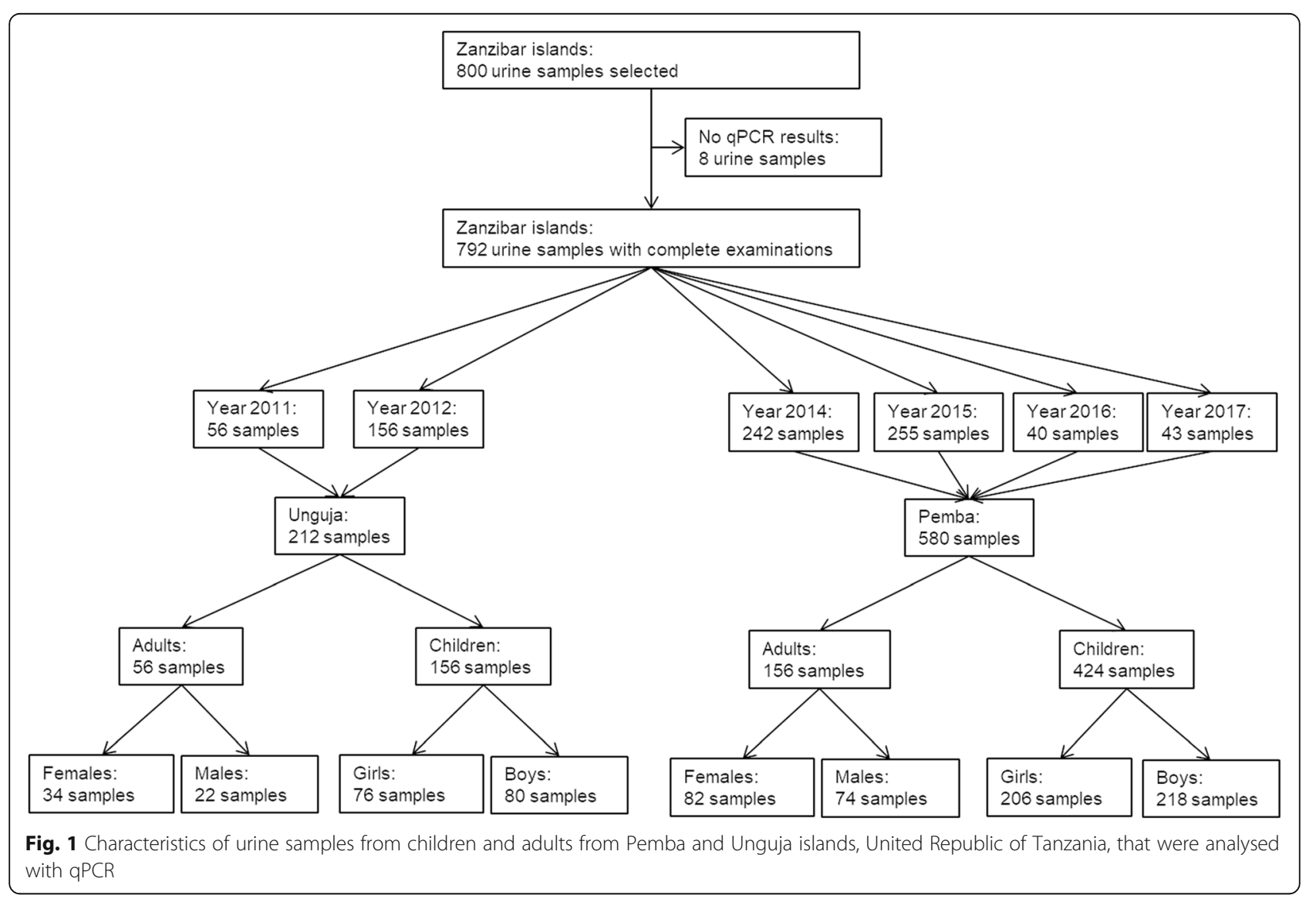


Table 2 Sensitivity and specificity of qPCR using urine filtration, reagent strips, or a combination of urine filtration and reagent strips as reference test for $S$. haematobium diagnosis in urine samples from Zanzibar

\begin{tabular}{|c|c|c|c|c|}
\hline & Urine filtration & Negative & Positive & Total \\
\hline \multirow[t]{6}{*}{ qPCR } & Negative & 569 & 11 & 580 \\
\hline & Positive & 118 & 94 & 212 \\
\hline & Total & 687 & 105 & 792 \\
\hline & Sensitivity & 89.5\% (95\% Cl: 87.4-91.7\%) & & \\
\hline & Specificity & $82.8 \%$ (95\% Cl: 80.2-85.5\%) & & \\
\hline & Reagent strips & Negative & Positive & Total \\
\hline \multirow[t]{6}{*}{ qPCR } & Negative & 557 & 23 & 580 \\
\hline & Positive & 126 & 86 & 212 \\
\hline & Total & 683 & 109 & 792 \\
\hline & Sensitivity & 78.9\% (95\% Cl: 76.1-81.7\%) & & \\
\hline & Specificity & 81.6\% (95\% Cl: 78.9-84.3\%) & & \\
\hline & Urine filtration plus reagent strips & Negative & Positive & Total \\
\hline \multirow[t]{5}{*}{ qPCR } & Negative & 553 & 27 & 580 \\
\hline & Positive & 110 & 102 & 212 \\
\hline & Total & 663 & 129 & 792 \\
\hline & Sensitivity & $79.1 \%$ (95\% Cl: $76.2-81.9 \%)$ & & \\
\hline & Specificity & $83.4 \%$ (95\% Cl: 80.8-86.0\%) & & \\
\hline
\end{tabular}

95\% Cl 95\% confidence interval

When the urine filtration was used as reference method, the qPCR identified Dra1 DNA in $89.5 \%$ (94/ $105)$ of $S$. haematobium egg-positive samples. As shown in Fig. 2 and Tab. S1, the probability of a positive Dra1 DNA result increased with increasing egg counts. The sensitivity of the qPCR was $80.6 \%(29 / 36)$ for counts between 1 and 5 eggs, 83.3\% (15/18) for counts between 6 and 10 eggs, $100 \%(23 / 23)$ for counts between 11 and 49 eggs and $96.4 \%(27 / 28)$ for counts of $50+$ eggs. The one egg-positive sample which was not identified as positive in the last group had a count of 721 eggs. With regard to egg-count infection intensity categorizations defined by the WHO, the sensitivity of qPCR to detect light and heavy infection intensities was $87.0 \%$ (67/77) and 96.4\% (27/28), respectively.

When reagent strips were used as reference method, the qPCR identified Dra1 DNA in 78.9\% (86/109) of microhaematuria-positive samples. The sensitivity for Dra1 DNA detection was higher when the microhaematuria grading was $++(81.5 \%, 22 / 27)$ and $+++(86.1 \%, 31 / 36)$, compared with trace $(75.0 \%, 21 / 28)$ and $+(66.7 \%, 12 / 18)$.

Compared with the combined results of urine filtration and reagent strips as reference method, the qPCR had a sensitivity of $79.1 \%$ and a specificity of $83.4 \%$.

\section{Characteristics of urine samples with false-negative qPCR results}

Tab. S2 shows the characteristics of the 27 urine samples that had S. haematobium egg counts in the urine filtration microscopy or microhaematuria-positive reagent strip results, but were negative for Dra1 DNA in the qPCR. Among them, 11 samples were identified as S. haematobium egg-positive by urine filtration but negative for Dra1 DNA by qPCR. Among the 11 samples, seven had egg counts ranging between 1 and 4 . When the microscope slides were read a second time for five among those seven slides, four had zero egg counts, but one among those four was microhaematuria-positive. Among these 11 samples, four had egg counts ranging between six and 721 eggs, mostly confirmed by a second reading of the microscope slides and all were microhaematuria-positive.

Among the 16 samples that were microhaematuriapositive but egg-negative and Dra1 DNA-negative, 13 were from females (three girls and 10 adult females).

\section{qPCR Ct-values as quantitative measure for infection intensity}

There was a significant negative correlation between Ctvalues measured by qPCR and S. haematobium eggs counted under the microscope (spearman's rho $=-0.49$, $P<0.001)$.

The median and first and third quartiles of Ct-values of samples when stratified by WHO defined infection intensity categories based on S. haematobium egg counts is illustrated in Fig. 3. In the egg-negative class, $75 \%$ of the Ct-values were $\geq 33$ with a median of 37 . In the light intensity egg-count class, $75 \%$ of the $\mathrm{Ct}$-values were $<31$ 


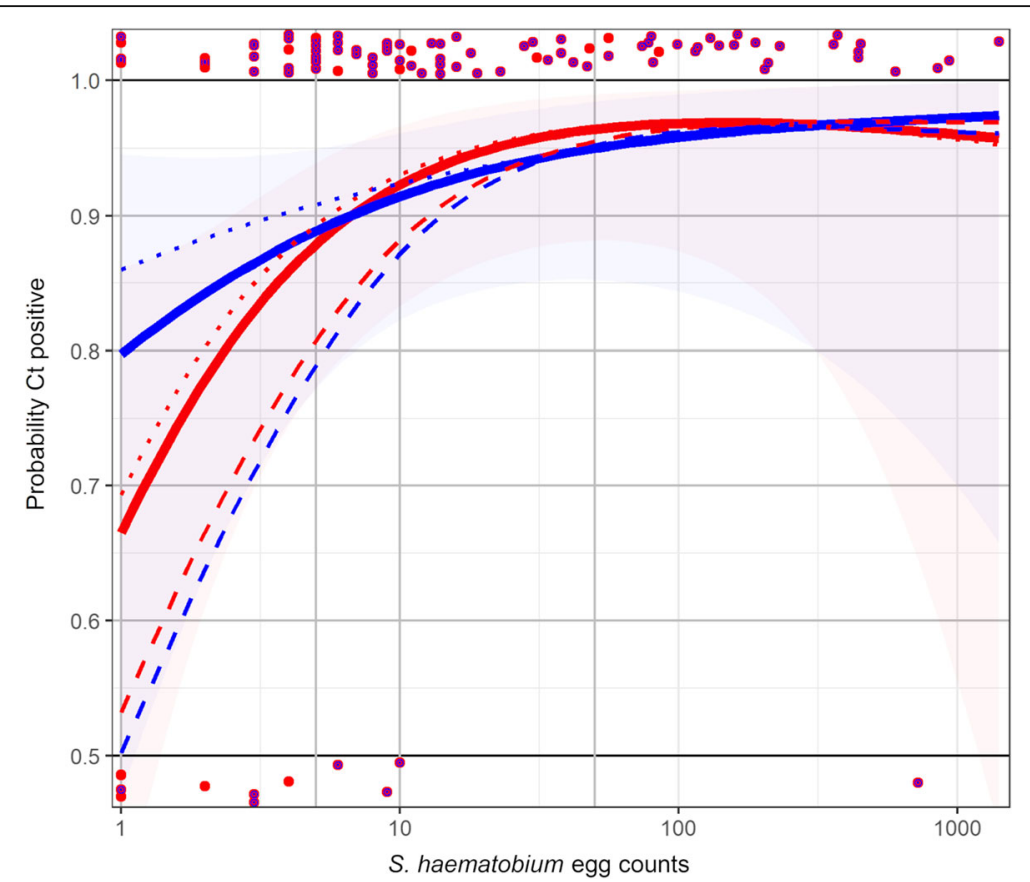

Fig. 2 Probability of a positive qPCR Ct-value in relation to increasing Schistosoma haematobium egg counts from urine filtration microscopy. Red dots: S. haematobium egg counts in qPCR-positive (top) or APCR-negative negative (bottom) samples, when Ct-values of $<33$ are considered as positive; red and blue dots: S. haematobium egg counts and microhaematuria in qPCR-positive or qPCR-negative negative samples, when Ctvalues of $<33$ are considered as positive; red line: probability curve for Ct-values $<33$ to become positive if urine filtration is the reference test; blue line: probability curve for Ct-values $<33$ to become positive if urine filtration plus reagent strip tests are the reference test; dotted red line: probability curve for Ct-values $<34$ to become positive if urine filtration is the reference test; dotted blue line: probability curve for Ct-values $<34$ to become positive if urine filtration plus reagent strip tests are the reference test; dashed red line: probability curve for Ct-values $<32$ to become positive if urine filtration is the reference test; dashed blue line: probability curve for Ct-values $<32$ to become positive if urine filtration plus reagent strip tests are the reference test

with a median of 26 . In the heavy intensity class, $75 \%$ of the Ct-values were $<24$ with a median of 21 . While the medians followed a decreasing trend, some overlap was observed.

\section{S. haematobium-positive rate in Zanzibar by qPCR}

The selection procedure for the 792 samples examined with qPCR was not designed to reveal a S. haematobium prevalence for Zanzibar in general or any of the study locations in particular. Figure $4 \mathrm{a}$ and $\mathrm{b}$ show the number of samples examined and the number of samples that were negative, egg-positive and/or Dral DNA-positive for each study site in Pemba and Unguja, respectively. A total of 223 egg-positive and/or Dra1 DNA-positive urine samples were identified in 34/46 locations in Pemba and in 30/45 locations in Unguja.

Among those 223 samples, 94 samples were recognized as $S$. haematobium-positive by both urine filtration and qPCR, 118 samples were diagnosed as positive by qPCR only, and 11 samples were identified by urine filtration only (Table 2). Among the 118 Dra1 DNA-positive only samples, 69 were samples from 23 sites in Pemba and 14 were samples from 5 sites in Unguja, where also additional, both eggpositive and Dra1 DNA-positive samples came from. The remaining 35 samples were from 16 locations in Unguja $(n=25)$ and nine locations in Pemba $(n=10)$, where no egg-positive samples were identified by urine filtration. Among the 11 egg-positive samples that were only identified by urine filtration but not qPCR, nine were from seven sites, where also additional egg-positive or Dra1 DNA-positive samples were identified and two samples were from one site in Pemba and Unguja each, where no other positives were found.

\section{Discussion}

The WHO envisions a world free of schistosomiasis [1]. Mass drug administration and complementary interventions to control and eliminate schistosomiasis in endemic areas have been scaled up over the past decades [5, 13, 24-26]. Moving towards elimination, sustained efforts will result in decreased Schistosoma prevalence and infection intensities [12, 27-29], and diagnosis of Schistosoma infections will become harder with standard classical parasitological methods [7]. Monitoring the 


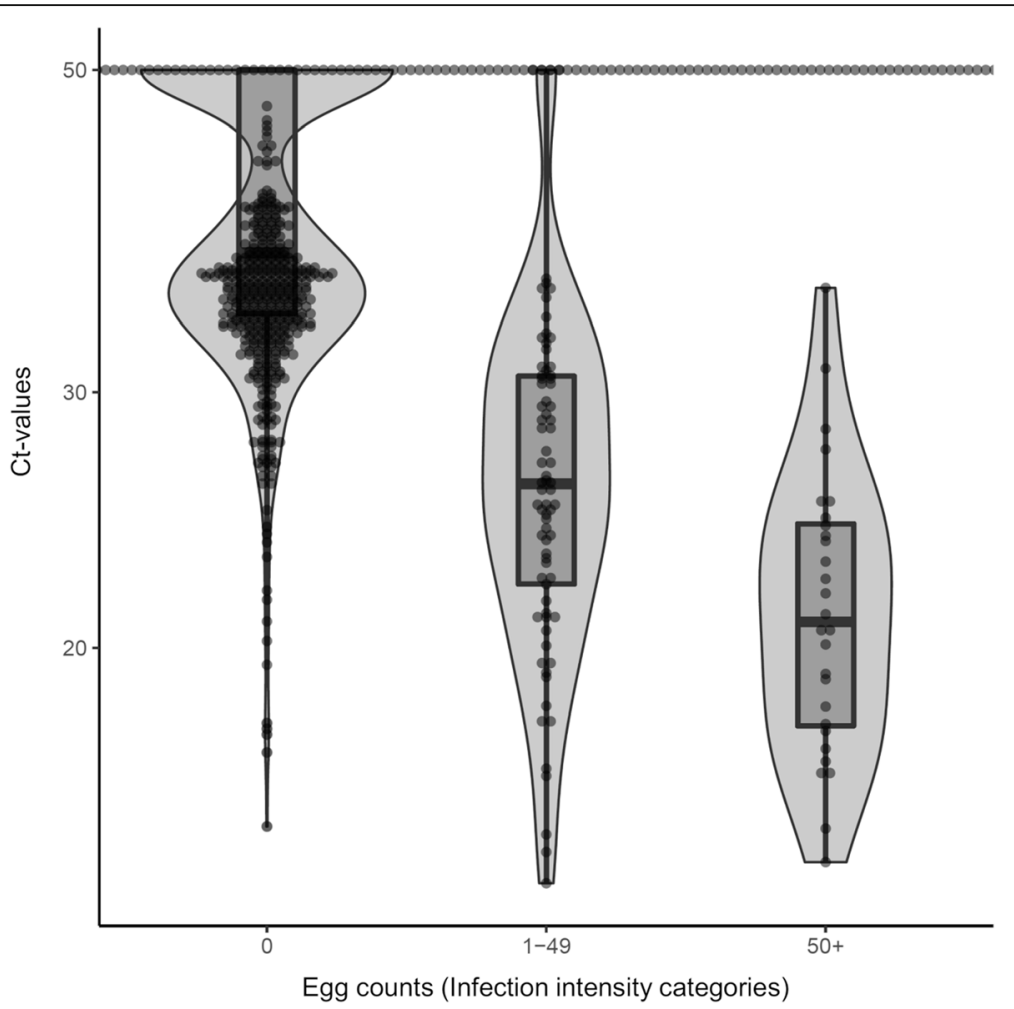

Fig. 3 qPCR Ct-values of samples stratified by infection intensity categories based on their $\mathrm{S}$. haematobium egg counts $(0$ eggs $=$ negative, 1-49 eggs $=$ light infection; $50+$ eggs $=$ heavy infection). Boxes represent 25,50 and 75\% quintiles. Whiskers present minimum and maximum without outliers

impact of interventions in elimination settings, surveillance to avoid recrudescence of infection, and verification of interruption of transmission require more sensitive, robust, and high-throughput approaches for S. haematobium diagnosis. Here, we assessed the performance of a standardized qPCR approach for $S$. haematobium diagnosis in a multi-laboratory ring trial and by examination of 792 urine samples collected in Zanzibar, one of the first settings in sub-Saharan Africa targeted for schistosomiasis elimination.

The ring-trial involving a total of eight research, hospital or biotech laboratories from Europe and Africa showed that all $S$. haematobium egg-positive samples were correctly identified by qPCR in all participating laboratories. Importantly, the results were comparable by running the qPCR on five different brands and types of thermocyclers. One laboratory obtained false-positive results in the egg-negative urine samples. Hence, while the standardized qPCR approach can be considered as accurately detecting $S$. haematobium-positive samples, the occurrence of false-positive samples could not be ruled out.

Applying the qPCR for $S$. haematobium diagnosis in urine samples from Zanzibar, Dra1 DNA detection by
qPCR clearly outperformed egg detection by urine filtration microscopy and microhaematuria assessment by reagent strips. The qPCR identified twice as many samples as $S$. haematobium-positive compared with the classical diagnostic methods. Most of the samples (108/118) that were Dra1 DNA-positive and S. haematobium eggnegative had Ct-values $<33$ and $>22$ and might hence be regarded as light intensity infections. While it is known that particularly very light intensity infections are often missed by reagent strips or urine filtration microscopy [7], it might also be that qPCR detects excreted parasite DNA in the absence of egg production. In that case, old egg granulomas might exist, which still release DNA. Or, worms could be present but not produce eggs at all or only intermittently. Circadian and daily variation in egg excretion have been reported for S. haematobium [30-32]. Hence, infection intensity classification based on egg counts from a single urine filtration from one day can be considered a meaningful measure at population level but not at individual level. Ct-levels from Schistosoma DNA have been reported to be more stable over multiple days than egg counts [32] and also our ring trial confirmed little variation between the different laboratories and technicians. Ct-values might therefore serve as a better measure of infection intensity than egg 


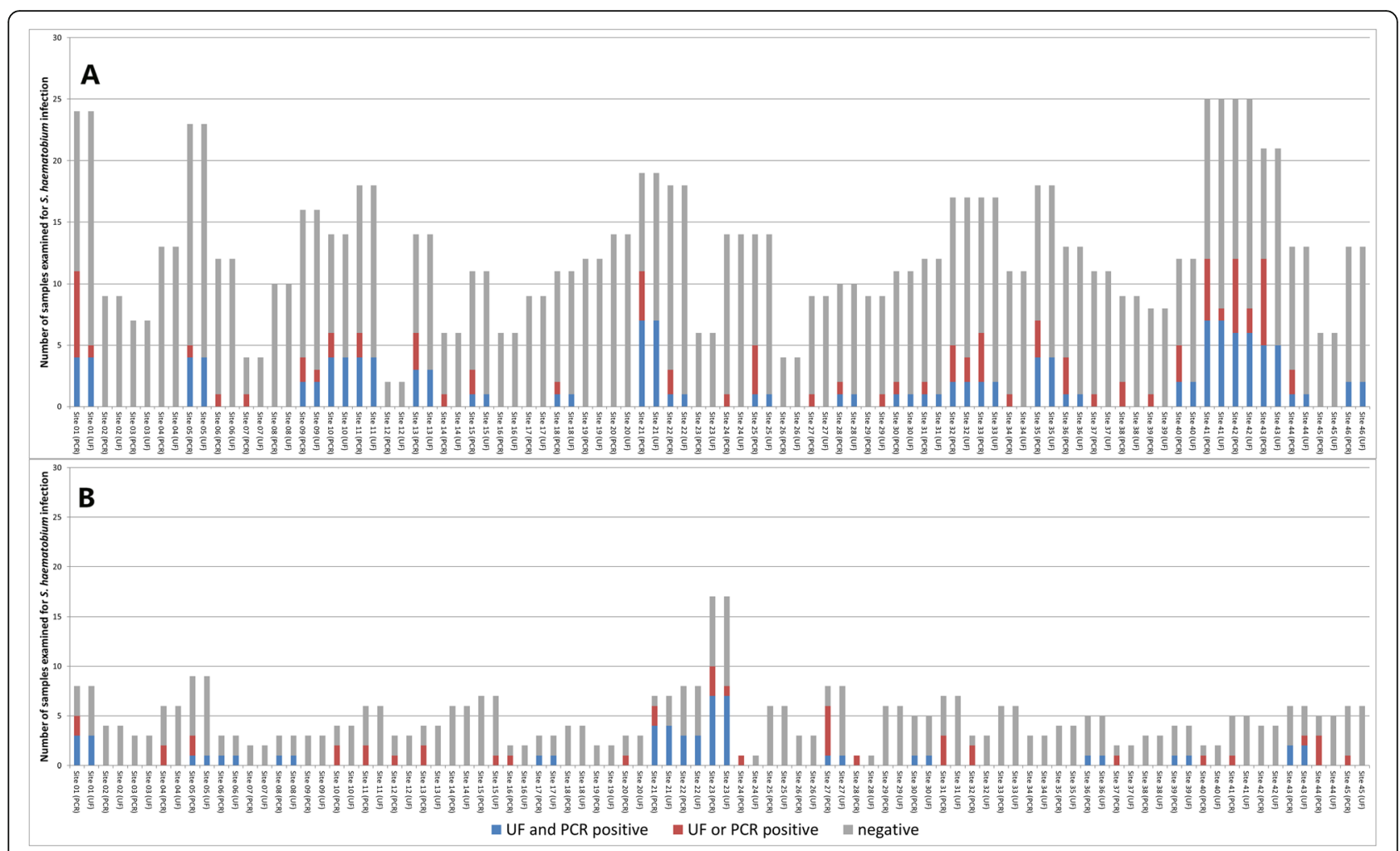

Fig. 4 Number of samples examined and identified as S. haematobium-positive with urine filtration (UF) and/or qPCR in 46 study sites in Pemba (a) and 45 study sites in Unguja (b) islands, United Republic of Tanzania

counts and might in future be used for evaluation of control and elimination programs as well as for drug efficacy assessment.

However, not all egg-positive and/or microhaematuriapositive urine samples were identified as Dra1 DNApositive. Most of these false-negative samples (7/11) had extremely low egg counts (below five eggs per $10 \mathrm{ml}$ urine) and some of them were not confirmed by quality control readings of the same slide. Hence, as suggested for falsenegative results in other studies, the amount of DNA in these very lightly infected samples might have been below the detectable limit $[32,33]$. However, four samples had counts between six and 721 eggs and it is not clear, why no DNA amplification occurred. There was no indication that storage time since sample collection, location of the participant's residence, sex or the microhaematuria level influenced the qPCR outcome in our study here, but labelling errors in 3\% of samples have been suggested as potential reason for discrepant diagnostic results in another study from Zanzibar working with urine samples from the same surveys [7]. Most of the microhaematuria-positive but egg-negative and Dra1 DNA-negative urine samples were from females, and the explanation that the microhaematuria was caused by menstruation blood or other infections of the urogenital tract is self- evident. Clearly, not all microhaematuria is caused by schistosomiasis [34].

Using urine filtration microscopy as reference method, the qPCR had a sensitivity of $89.5 \%$. This value is greatly in line with previous assessments of qPCR systems for S. haematobium [32, 33, 35, 36]. Also the sensitivity of our qPCR approach for light (87.0\%) and heavy (96.4\%) intensity infections matches very well with the sensitivity revealed by Obeng et al. [33]. Going beyond the WHO classifications, we found that the sensitivity was 80.6 and $83.3 \%$ at egg counts of $1-5$ and $6-10$ eggs per $10 \mathrm{ml}$ urine, respectively, and that the sensitivity reached (almost) $100 \%$ at counts $>10$ eggs per $10 \mathrm{ml}$.

The examination of urine samples from 91 study sites in Zanzibar showed that the qPCR mostly identified additional S. haematobium-positive individuals in locations, where also the urine filtration microscopy identified cases. Hence, in these places the S. haematobium prevalence is likely underestimated when urine filtration is used as diagnostic approach, and might be twice as high as assumed. Moreover, the qPCR identified some few cases in sites where all urine filtration examinations were negative. Both observations are in line with an earlier study using the highly sensitive up-converting phosphor lateral flow circulating anodic antigen (UCP-LF CAA) 
assay in Pemba, which showed that there are far more individuals infected with $S$. haematobium than estimated by the urine filtration [11]. The results have important ramifications for future elimination efforts on the islands. Clearly, the true prevalence is considerably higher than the apparent prevalence and schistosomiasis elimination interventions need to be sustained to avoid a rebound of infections and to advance the gains that have been made in Zanzibar to date. Mass drug administration to further reduce the $S$. haematobium prevalence might be justified for an extended period, at least in areas where urine filtration shows a reasonably high prevalence (and the true prevalence might again be higher). If, as suggested elsewhere [12, 13], the Zanzibar schistosomiasis elimination programme, guided by donor decisions and research outcomes based on urine filtration, considers changing the tactics from MDA to more tailored intervention approaches such as surveillance-response including test and treat approaches, a sensitive diagnosis should be warranted. Ideally, the new diagnostic test should be applicable at the point of care, i.e. not only in a well-equipped central laboratory, but also at the peripheral level in health facilities, hospitals, and within control and elimination programs in basic laboratories, schools and households. It should also be rapid and high-throughput so that infected individuals can be tested at the spot and treated immediately if positive. DNA-based tests with point-ofcare application include the loop-mediated isothermal amplification (LAMP) $[37,38]$ and the recently developed recombinase polymerase amplification (RPA) [39] assays. The qPCR approach used in this study is not a rapid diagnostic test that can be used at peripheral level in facilities without additional equipment and electricity. It requires a well-equipped laboratory, experienced and organized technicians, and a considerable amount of time, particularly for DNA extraction, if many samples are to be tested. If not for routine surveillance in endemic countries, qPCR, in an improved format and with further increased sensitivity and specificity so that also ultra-light intensity infections are reliably detected, may yet be the approach of choice for verification and certification of elimination, once successful schistosomiasis elimination programmes need the proof of interruption of transmission. Moreover, a standardized and highly sensitive and specific qPCR approach will be highly useful to measure the impact of control and elimination interventions in low-prevalence settings in endemic countries where it will be able to more accurately assess the prevalence and incidence levels than classical parasitological methods. Also, qPCR might have a role to play in the detection of female genital schistosomiasis when urine egg counts are low. Finally, the application of qPCR can be recommended for a clinical environment in Europe and the United States, where individual diagnosis is important and where migrants, travellers and tourists may present with very light intensity or prepatent infections that may be missed by classical parasitological diagnosis.

\section{Conclusions}

The qPCR approach presented here is a standardized and sensitive approach for $S$. haematobium diagnosis that can be used for impact assessment of schistosomiasis elimination programmes, individual diagnosis of $S$. haematobium infection, and in improved format also for verification and certification of elimination.

\section{Supplementary information}

Supplementary information accompanies this paper at https://doi.org/10. 1186/s40249-020-00726-y.

Additional file 1: Table S1. showing the multivariate frequency distribution of S. haematobium infection measured by urine filtration (egg counts), reagent strips (microhaematuria), and qPCR (Dra1 DNA).

Additional file 2: Table S2. showing the characteristics of 27 urine samples that had S. haematobium egg counts in the urine filtration (UF) microscopy or microhaematuria-positive reagent strip results, but were negative for Dral DNA in the GPCR.

\section{Abbreviations}

MDA: Mass drug administration; MoH: Ministry of Health; PHL-IdC: Public Health Laboratory-Ivo de Carneri; qPCR: Real time-polymerase chain reaction; WHO: World Health Organization; ZEST: Zanzibar Elimination of

Schistosomiasis Transmission

\section{Acknowledgements}

We thank the staff of the parasitology teams in Unguja and Pemba who helped with the collection and examination of urine samples, subjected to haematuria testing and urine filtration. We are grateful to the eight

laboratories from Europe and Africa who participated in the ring trial: Ifakara Health Institute, Bagamoyo Research and Training Centre, Bagamoyo, United Republic of Tanzania; Inqaba Biotechnical Industries (Pty) Ltd., Pretoria, South Africa; Leiden University Medical Centre, Leiden, the Netherlands; Natural History Museum, London, United Kingdom; Norwegian Veterinary Institute, Oslo, Norway; Saarland University Medical Center, Institute of Medical Microbiology and Hygiene, Homburg, Germany; Swiss Tropical and Public Health Institute, Basel, Switzerland.

\section{Authors' contributions}

Conception and design of the study: DK, CS, CD, FA, RS, and SK. Acquisition of data: DK, JR, JD, SMAm, SMAI, FK, DR and SK. Analysis and interpretation of data: DK, CS, JH, and SK. Drafting the article: SK. Revising the article critically for important intellectual content: DK, JR, JD, CS, CD, FA, SMAm, SMAI, FK, JH, DR, RS and SK. All authors read and approved the final manuscript.

\section{Funding}

This study received financial support from Innosuisse (project 18553.2 PFLSLS) and from the University of Georgia Research Foundation Inc., which is funded by the Bill \& Melinda Gates Foundation for the Schistosomiasis Consortium for Operational Research and Evaluation (SCORE) projects (prime award no. 50816, sub-award no. RR374-053/4893206). SK received financial support by sub-award no. RR374-053/4893196 and via direct grants from the Bill \& Melinda Gates Foundation (Investment IDs: OPP1191423 and OPP1198086). FA received financial support from the Wellcome Trust (SCAN Project 104958/Z/14/Z). These funders had no role in study design, data collection and analysis, decision to publish, or preparation of the manuscript. As industry partner of the Innosuisse project, Biolytix AG supported the study by designing and implementing the GPCR approach, and by providing 
equipment, material, and personnel for the analyses of samples. Biolytix AG contributed to data collection and the preparation of the manuscript.

\section{Availability of data and materials}

The data supporting the conclusions of this article are included within the article and its additional files. The datasets generated and/or analysed during the current study are available from the corresponding author upon reasonable request.

\section{Ethics approval and consent to participate}

Our study protocol for sample collection in Zanzibar was approved by the Zanzibar Medical Research Ethics Committee (ZAMREC) in Zanzibar, United Republic of Tanzania (reference no. ZAMREC 0003/Sept/011), the Ethics Committee of Basel, Switzerland (reference no. EKBB 236/11), and the Institutional Review Board of the University of Georgia in the USA (project no. 2012-10138-0). The study is registered with the International Standard Randomized Controlled Trial Number register (ISRCTN48837681). All children and adults invited to participate received information about the study. Only individuals with written informed consent were included in the study. For children, the consent form had to be signed by their parents or their legal guardians. All study participants were offered praziquantel $(40 \mathrm{mg} / \mathrm{kg})$ against schistosomiasis free of charge via the biannual island-wide mass drug administration campaigns conducted as part of the elimination interventions by the Zanzibar Neglected Diseases Programme from 2012 till 2017.

\section{Consent for publication}

Not applicable.

\section{Competing interests}

The authors declare financial and non-financial competing interests as follows:

Biolytix AG is a commercial entity that may gain or lose financially from the publication of the manuscript. Authors from the Swiss Tropical and Public Health Institute, the Zanzibar Neglected Diseases Programme, the Public Health Laboratory-Ivo de Carneri and the Natural History Museum received support for the here presented research in terms of collaboration and nonmonetary support in terms of facilities, equipment, material and research personnel from Biolytix AG.

\section{Author details}

'Biolytix AG, Benkenstrasse 254, 4108 Witterswil, Switzerland. ${ }^{2}$ Swiss Tropical and Public Health Institute, Socinstrasse 57, 4002 Basel, Switzerland. ${ }^{3}$ University of Basel, Petersplatz 1, 4001 Basel, Switzerland. ${ }^{4}$ Wolfson Wellcome Biomedical Laboratories, Department of Life Sciences, Natural History Museum, Cromwell Road, London SW7 5BD, UK. ${ }^{5}$ Public Health Laboratory Ivo de Carneri, P.O. Box 122, Chake-Chake, Pemba, United Republic of Tanzania. ${ }^{6}$ Neglected Diseases Programme, Ministry of Health, P.O. Box 236, Zanzibar Town, Unguja, United Republic of Tanzania.

\section{Received: 27 March 2020 Accepted: 14 July 2020}

\section{Published online: 04 September 2020}

\section{References}

1. WHO. Schistosomiasis: progress report 2001-2011 and strategic plan 20122020. Geneva: World Health Organization; 2013.

2. GBD 2017 DALYs and HALE Collaborators. Global, regional, and national disability-adjusted life-years (DALYS) for 359 diseases and injuries and healthy life expectancy (HALE) for 195 countries and territories, 1990-2017: a systematic analysis for the Global Burden of Disease Study 2017. Lancet. 2018;392(10159):1859-922.

3. WHO. Accelerating work to overcome the global impact of neglected tropical diseases - a roadmap for implementation. Geneva: World Health Organization; 2012.

4. WHO. WHA65.21. Elimination of schistosomiasis. Sixty-fifth World Health Assembly Geneva 21-26 May 2012 Resolutions, decisions and annexes. WHA65/2012/REC/1. Geneva: World Health Organization; 2012. p. 36-7.

5. WHO. Schistosomiasis and soil-transmitted helminthiases: number of people treated in 2017. Week Epid Rec. 2018;50(93):681-92.

6. Rollinson D, Knopp S, Levitz S, Stothard JR, Tchuem Tchuenté LA, Garba A, et al. Time to set the agenda for schistosomiasis elimination. Acta Trop. 2013;128(2):423-40.
7. Knopp S, Ame SM, Hattendorf J, Ali SM, Khamis IS, Bakar F, et al. Urogenital schistosomiasis elimination in Zanzibar: accuracy of urine filtration and haematuria reagent strips for diagnosing light intensity Schistosoma haematobium infections. Parasit Vectors. 2018;11(1):552

8. Oliveira WJ, Magalhaes FDC, Elias AMS, de Castro VN, Favero V, Lindholz CG, et al. Evaluation of diagnostic methods for the detection of intestinal schistosomiasis in endemic areas with low parasite loads: saline gradient, Helmintex, Kato-Katz and rapid urine test. PLoS Negl Trop Dis. 2018;12(2): e0006232.

9. Bergquist $\mathrm{R}$, Johansen MV, Utzinger J. Diagnostic dilemmas in helminthology: what tools to use and when? Trends Parasitol. 2009;25(4): $151-6$.

10. Bergquist R, Zhou XN, Rollinson D, Reinhard-Rupp J, Klohe K. Elimination of schistosomiasis: the tools required. Infect Dis Poverty. 2017;6(1):158.

11. Knopp S, Corstjens PL, Koukounari A, Cercamondi Cl, Ame SM, Ali SM, et al. Sensitivity and specificity of a urine circulating anodic antigen test for the diagnosis of Schistosoma haematobium in low endemic settings. PLoS Negl Trop Dis. 2015;9(5):e0003752.

12. Knopp S, Ame SM, Person B, Hattendorf J, Rabone M, Juma S, et al. A 5-year intervention study on elimination of urogenital schistosomiasis in Zanzibar: parasitological results of annual cross-sectional surveys. PLoS Negl Trop Dis. 2019;13(5):e0007268.

13. Knopp S, Person B, Ame SM, Ali SM, Hattendorf J, Juma S, et al. Evaluation of integrated interventions layered on mass drug administration for urogenital schistosomiasis elimination: a cluster-randomised trial. Lancet Glob Health. 2019;7(8):e1118-e29.

14. Knopp S, Mohammed KA, Ali SM, Khamis IS, Ame SM, Albonico M, et al. Study and implementation of urogenital schistosomiasis elimination in Zanzibar (Unguja and Pemba islands) using an integrated multidisciplinary approach. BMC Public Health. 2012;12(1):930.

15. OCGS. Zanzibar statistical abstract 2018. Zanzibar: Office of the Chief Government Statistician; 2019.

16. McCullough FS, Krafft JG. Schistosomiasis in Zanzibar and Pemba. Report on a mission 1 April - 7 June 1975. Geneva: World Health Organization; 1976.

17. Savioli L, Dixon H, Kisumku UM, Mott KE. Control of morbidity due to Schistosoma haematobium on Pemba island: programme organization and management. Trop Med Parasitol. 1989;40(2):189-94.

18. Mgeni AF, Kisumku UM, McCullough FS, Dixon H, Yoon SS, Mott KE. Metrifonate in the control of urinary schistosomiasis in Zanzibar. Bull World Health Organ. 1990;68(6):721-30.

19. Stothard JR, French MD, Khamis IS, Basáñez MG, Rollinson D. The epidemiology and control of urinary schistosomiasis and soil-transmitted helminthiasis in schoolchildren on Unguja island, Zanzibar. Trans R Soc Trop Med Hyg. 2009;103(10):1031-44.

20. Guidi A, Andolina C, Ame SM, Albonico M, Cioli D, Juma HH. Praziquantel efficacy and long-term appraisal of schistosomiasis control in Pemba island. Tropical Med Int Health. 2010;15(5):614-8.

21. Knopp S, Person B, Ame SM, Mohammed KA, Ali SM, Khamis IS, et al. Elimination of schistosomiasis transmission in Zanzibar: baseline findings before the onset of a randomized intervention trial. PLoS Negl Trop Dis. 2013;7:e2474.

22. Hamburger J, He N, Abbasi I, Ramzy RM, Jourdane J, Ruppel A. Polymerase chain reaction assay based on a highly repeated sequence of Schistosoma haematobium: a potential tool for monitoring schistosome-infested water. Am J Trop Med Hyg. 2001;65(6):907-11.

23. Cnops L, Soentjens P, Clerinx J, Van Esbroeck M. A Schistosome haematobium-specific real-time PCR for diagnosis of urogenital schistosomiasis in serum samples of international travelers and migrants. PLoS Negl Trop Dis. 2013;7(8):e2413.

24. Zou L, Ruan S. Schistosomiasis transmission and control in China. Acta Trop. 2015;143:51-7.

25. Utzinger J, Zhou XN, Chen MG, Bergquist R. Conquering schistosomiasis in China: the long march. Acta Trop. 2005;96(2-3):69-96.

26. Sun LP, Wang W, Hong QB, Li SZ, Liang YS, Yang HT, et al. Approaches being used in the national schistosomiasis elimination programme in China: a review. Infect Dis Poverty. 2017;6(1):55.

27. Phillips AE, Gazzinelli-Guimaraes PH, Aurelio HO, Ferro J, Nala R, Clements M et al. Assessing the benefits of five years of different approaches to treatment of urogenital schistosomiasis: a SCORE project in northern Mozambique. PLoS Negl Trop Dis. 2017;11(12):e0006061.

28. Shen $\mathrm{Y}$, Wiegand RE, Olsen A, King $\mathrm{CH}$, Kittur N, Binder S, et al. Five-year impact of different multi-year mass drug administration strategies on 
childhood Schistosoma mansoni-associated morbidity: a combined analysis from the Schistosomiasis consortium for operational research and evaluation cohort studies in the Lake Victoria regions of Kenya and Tanzania. Am J Trop Med Hyg. 2019:101(6):1336-44.

29. NTD Modelling Consortium Schistosomiasis Group. Insights from quantitative and mathematical modelling on the proposed WHO 2030 goal for schistosomiasis [version 2; peer review: 2 approved, 1 approved with reservations]. Gates Open Res. 2019;3:1517.

30. Doehring E, Feldmeier H, Daffalla AA. Day-to-day variation and circadian rhythm of egg excretion in urinary schistosomiasis in the Sudan. Ann Trop Med Parasitol. 1983;77(6):587-94.

31. Van Etten L, Kremsner PG, Krijger FW, Deelder AM. Day-to-day variation of egg output and schistosome circulating antigens in urine of Schistosoma haematobium-infected school children from Gabon and follow-up after chemotherapy. Am J Trop Med Hyg. 1997:57(3):337-41.

32. Vinkeles Melchers NV, van Dam GJ, Shaproski D, Kahama Al, Brienen EA, Vennervald BJ, et al. Diagnostic performance of Schistosoma real-time PCR in urine samples from Kenyan children infected with Schistosoma haematobium: day-to-day variation and follow-up after praziquantel treatment. PLoS Negl Trop Dis. 2014;8(4):e2807.

33. Obeng BB, Aryeetey YA, de Dood CJ, Amoah AS, Larbi IA, Deelder AM, et al. Application of a circulating-cathodic-antigen (CCA) strip test and real-time $\mathrm{PCR}$, in comparison with microscopy, for the detection of Schistosoma haematobium in urine samples from Ghana. Ann Trop Med Parasitol. 2008; 102(7):625-33.

34. Krauth SJ, Greter H, Stete K, Coulibaly JT, Traore SI, Ngandolo BN, et al. All that is blood is not schistosomiasis: experiences with reagent strip testing for urogenital schistosomiasis with special consideration to very-low prevalence settings. Parasit Vectors. 2015;8:584

35. Ibironke O, Koukounari A, Asaolu S, Moustaki I, Shiff C. Validation of a new test for Schistosoma haematobium based on detection of Dra1 DNA fragments in urine: evaluation through latent class analysis. PLoS Negl Trop Dis. 2012;6(1):e1464.

36. Aryeetey YA, Essien-Baidoo S, Larbi IA, Ahmed K, Amoah AS, Obeng BB, et al. Molecular diagnosis of Schistosoma infections in urine samples of school children in Ghana. Am J Trop Med Hyg. 2013;88(6):1028-31.

37. Gandasegui J, Fernandez-Soto P, Dacal E, Rodriguez E, Saugar JM, Yepes E, et al. Field and laboratory comparative evaluation of a LAMP assay for the diagnosis of urogenital schistosomiasis in Cubal, Central Angola. Tropical Med Int Health. 2018;23(9):992-1001.

38. Lodh N, Mikita K, Bosompem KM, Anyan WK, Quartey JK, Otchere J, et al. Point of care diagnosis of multiple schistosome parasites: species-specific DNA detection in urine by loop-mediated isothermal amplification (LAMP), Acta Trop. 2017;173:125-9.

39. Rostron P, Pennance T, Bakar F, Rollinson D, Knopp S, Allan F, et al. Development of a recombinase polymerase amplification (RPA) fluorescence assay for the detection of Schistosoma haematobium. Parasit Vectors. 2019;12(1):514

Ready to submit your research? Choose BMC and benefit from:

- fast, convenient online submission

- thorough peer review by experienced researchers in your field

- rapid publication on acceptance

- support for research data, including large and complex data types

- gold Open Access which fosters wider collaboration and increased citations

- maximum visibility for your research: over $100 \mathrm{M}$ website views per year

At $\mathrm{BMC}$, research is always in progress.

Learn more biomedcentral.com/submissions 